\title{
Comparison of Three Surgical Techniques in Pilonidal Sinus Surgery
}

\author{
Abdulcabbar Kartal', Hüseyin Onur Aydın², Mehmet Oduncu ${ }^{3}$, \\ Murat Ferhat Ferhatoğlu', Taner Kıvılcım', Ali ilker Filiz ${ }^{1}$ \\ ${ }^{1}$ Department of General Surgery, Okan University Medicine Faculty, Istanbul, \\ Turkey; \\ ${ }^{2}$ Department of General Surgery, Başkent University Medicine Faculty, Ankara, \\ Turkey; \\ ${ }^{3}$ Department of General Surgery, Defne Hospital, Antakya, Turkey
}

Received August 23, 2018; Accepted January 31, 2019.

Key words: Pilonidal sinus - Perforator flap - Rhomboid flap - Complication Recurrence

Abstract: Our study aims to compare the surgical outcome of Limberg transposition flap, Karydakis flap, and primary closure after excision to treat sacrococcygeal pilonidal sinus disease. A total of 634 patients with pilonidal sinus who underwent surgery were evaluated retrospectively from January 2014 to January 2016. The patients were divided into three groups. Limberg transposition flap (LTF) was performed in 131 patients (group 1), Karydakis flap (KF) was performed in 232 patients (group 2) and primary closure (PC) after excision was performed in 271 patients (group 3). Patient demographics, operative and postoperative outcomes were recorded and analyzed retrospectively. The mean age $(p=0.98)$, sex ratio $(p=0.74)$ and removed sinus volume $(p=0.67)$ were not statistically different between groups. Mean operative time was $54.3 \pm 6.4 \mathrm{~min}$ for group 1, $46.8 \pm 10.5 \mathrm{~min}$ for group 2 , and $26.9 \pm 5.8$ min for group 3 respectively $(p=0.01)$. When the length of hospital stay was compared, there was a significant difference in favor of primary closure $(p=0.01)$. Regarding early surgical complication, Karydakis flap technique was superior to other groups $(p<0.001)$. The recurrent rate was higher in the primary closure group $(p<0.001)$. In our study, the primary closure method regarding the duration of surgery and hospitalization; Karydakis method regarding postoperative complications (seroma, hematoma, wound dissociation, infection, recurrence) were superior to the other two methods.

Mailing Address: Abdulcabbar Kartal, MD., i̇çmeler mah. Aydınlı yolu cad., Aydemir sok., No. 2, Tuzla, Istanbul, Turkey; Phone: 009021649465 26; e-mail: abdulcabbar.kartal@okan.edu.tr 


\section{Introduction}

Pilonidal sinus disease (PSD) is a chronic inflammation of the natal cleft and is mainly seen in young adults (Allen-Mersh, 1990; Hull and Wu, 2002). PSD usually occurs in healthy young men (male/female $=4-5 / 1)$. The incidence of the disease is approximately 26 per 100,000 people, with a mean age of 19 years for women and 21 years for men (Gurer et al., 2005). Although some earlier works have regarded the condition as congenital, it has recently become widely accepted that the condition is acquired (Bascom, 1983). Etiology of this disease is uncertain but relates to the implantation of loose hair into the depth of the natal cleft. Factors that affect the condition are the nature of hairs, the force of implantation and vulnerability of the skin (Shabbir et al., 2014). The goals in pilonidal sinus disease surgery are rapid healing, minimal hospital admission, minimal patient inconvenience, and low recurrence. There is no method satisfies all the requirements for the ideal treatment. The management of pilonidal disease depends on its presentation and ranges from simple incision and drainage to a wide excision with extensive reconstructive procedures (Varnalidis et al., 2014). Although many surgical procedures have been tried, the best surgical method remains controversial because of high recurrence rates. The recurrence rate after surgical treatment of PSD varies from 3 to $46 \%$ (Shafik, 1996).

There is no consensus on the optimal surgical management of the pilonidal sinus disease. Our objective is to compare the three methods used by our clinic and determine the outcomes retrospectively about operative time, hospitalization time, healing, postoperative complications, and recurrence.

\section{Material and Methods}

Between January 2014 and January 2016, 670 patients (Figure 1) underwent surgery for PSD in the Department of General Surgery at Siverek Government Hospital in Sanliurfa and Okan University Hospital in Istanbul. The data were collected after receiving permission from the hospital administration. A statement of informed consent was obtained from all patients before the operation. The patients who had previous PSD surgery $(n=23)$ and concurrent abscess formation $(n=13)$ were excluded from the study (Figure 1). The diagnosis of recurrent PSD was made based on clinical features. The remaining 634 patients who underwent surgery comprised the study groups and were evaluated retrospectively. All operations were performed by general surgery specialists with at least five years of experience. According to the types of surgeries that performed, patients were randomly divided into three groups. Patients who underwent Limberg transposition flap (LTF) were included in group 1, Karydakis flap (KF) were included in group 2 and excision, and primary closure (PC) were included in group 3, respectively. Patients with multiple sinus orifices in the sacrococcygeal region and the perianal region with a sinus opening less than $3 \mathrm{~cm}$ in length included in the KF group. Patients with more than three sinus orifice in the sacrococcygeal region and has sentinel sinus orifice $3 \mathrm{~cm}$ in the 


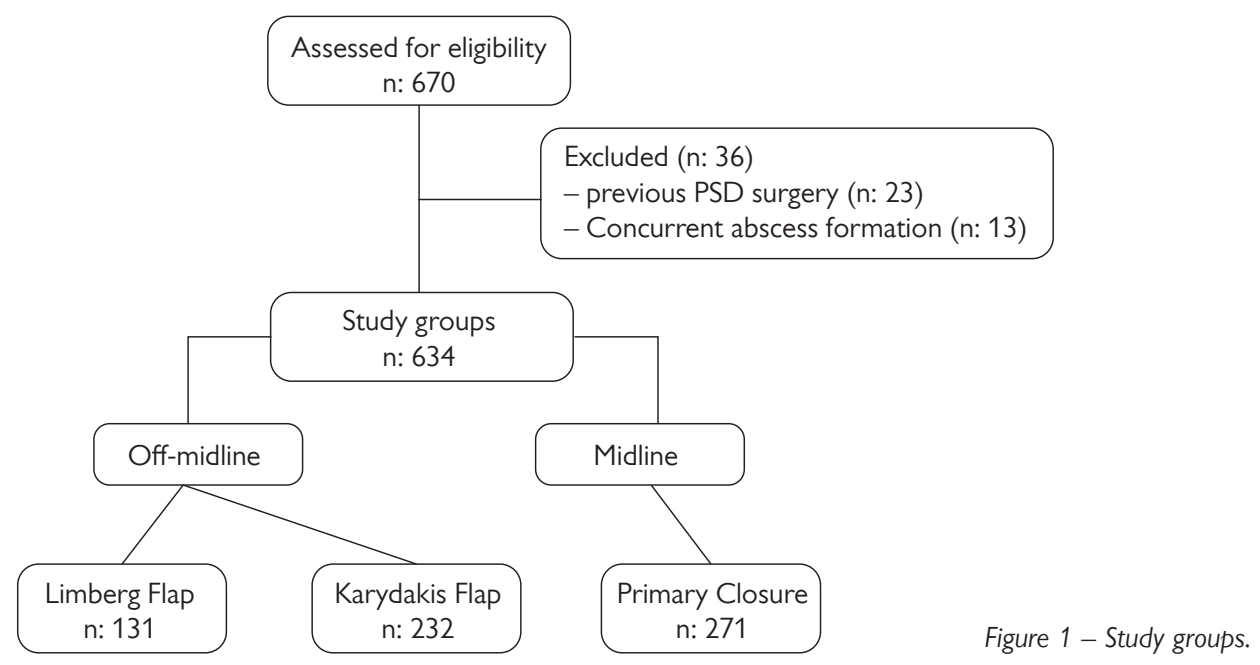

distance from the sinus mouths included LTF group and patients with 3 or less sinus orifice in the sacrococcygeal region was preferred in PC group. In the study, age and gender of patients, type and duration of operation and length of hospitalization were evaluated. Postoperative early complications (seroma, hematoma, wound dehiscence, infection) and recurrence rates were compared between groups. The term "recurrence" is defined as fistula formation, wound dehiscence with abscess and purulent drainage from incision line in the first year after the operation.

\section{Surgical technique}

Hair of the gluteal and sacral regions was shaved before the operation. Preoperative bowel preparation was not used. In all cases, prophylactic antibiotics (cefazolin sodium at a dose of $1 \mathrm{~g}$ was given intravenously) were used at the time of induction of anesthesia. Patients were operated under Jack Knife position with spinal anesthesia. The gluteal skin was retracted toward to both sides using adhesive bandages to expose the intergluteal sulcus, and then methylene blue was administered in the sinus opening at the gluteal area. Excision of all sinus tracts, fistula borders, and scar tissues was carried out deep to the postsacral fascia and surrounding undamaged fibrolipomatous tissues. The volume of each piece removed was measured using a beaker and physiological saline. The volume of the overflowing physiological saline was taken as the total piece volume. Hemovac drain was used in patients with extracted sinus volume of $20 \mathrm{cc}$ and above.

In LTF group, after removing the specimen, the Limberg fasciocutaneous flap was prepared by extending the incision down to and through the right or left gluteus maximus fascia (Varnalidis et al., 2014). The flap was sutured to the presacral fascia using 2/0 vicryl suture material. Subcutaneous tissues were approached 
with $2 / 0$ vicryl sutures. Skin closure was accomplished using $2 / 0$ prolene mattress sutures.

In KF group, Karydakis flap extending along the incision was prepared with the medial edge of the wound being $1 \mathrm{~cm}$ deep and extending $2-3 \mathrm{~cm}$ medially (interiorly). The flap was displaced medially and sutured to the presacral fascia using $2 / 0$ vicryl suture material. Subcutaneous tissues were approached with 2/0 vicryl sutures. Skin closure was accomplished using 2/0 prolene mattress sutures (Shafik, 1996).

In PC group, after removing the specimen, subcutaneous tissues were approached with no. 1 vicryl sutures. Skin closure was accomplished using 2/0 prolene mattress sutures.

All patients were released for mobilization on the first postoperative day. The suction drains were removed when daily drainage below $20 \mathrm{ml}$ was obtained. In general, the patients were discharged on the first or second postoperative day for PC group and the third or fourth postoperative day for flap groups. The sutures were removed on the tenth postoperative day. The patients were advised to avoid pressure on the operated site for 10-15 days postoperatively. All cases were examined twice during the first two weeks, and once at the end of 1, 3, and six months after the operation, and yearly thereafter. The mean follow-up was 28 (22-35) months.

Statistical analyses were performed using SPSS (Statistical Package for the Social Sciences ver. 20.0, SPSS Inc., Chicago, Illinois, USA) computer software. One-way ANOVA test was used to compare the duration of hospital stay and operative time between the groups. Complications (seroma, hematoma, wound dehiscence, infection, recurrence) were analyzed using the chi-square test. Results were given as mean \pm standard deviation. For all statistical analyses, $p<0.05$ was considered significant.

\section{Results}

A total of 634 patients (Figure 1) were included in the study (LTF: 131, KF: 232, PC: 271$)$ and 36 patients were excluded due to previous PDS surgery $(n=23)$ and

\section{Table 1 - The demographic datas of the patients}

\begin{tabular}{lcccc}
\hline Charecteristics & Limberg flap & Karydakis flap & Primary closure & P-value \\
\hline Numbers $(\mathrm{n})$ & 131 & 232 & 271 & \\
Gender (male/female) & $25 / 106$ & $42 / 190$ & $58 / 213$ & 0.740 \\
Age (years) & $26.31 \pm 8.40$ & $26.39 \pm 7.34$ & $26.44 \pm 7.11$ & 0.980 \\
Hospitalization (days) & $3.31 \pm 0.87$ & $2.10 \pm 0.73$ & $1.71 \pm 1.48$ & 0.001 \\
Operating time (min) & $54.31 \pm 6.41$ & $46.85 \pm 10.46$ & $26.94 \pm 5.79$ & 0.001 \\
Specimen volume (cc) & $28 \pm 52$ & $29 \pm 51$ & $28 \pm 65$ & 0.670 \\
\hline
\end{tabular}

Values are mean \pm standard deviation (range) 
Table 2 - Postoperative outcomes and recurrence

\begin{tabular}{lccccc}
\hline Complications & $\begin{array}{c}\text { Limberg } \\
\text { flap }\end{array}$ & $\begin{array}{c}\text { Karydakis } \\
\text { flap }\end{array}$ & $\begin{array}{c}\text { Primary } \\
\text { closure }\end{array}$ & Total & P-value \\
\hline Seroma $(\mathrm{n} / \%)$ & $13 / 9.92 \%$ & $10 / 4.31 \%$ & $41 / 15.13 \%$ & $64 / 10.09 \%$ & $<0.001$ \\
Wound dehiscence $(\mathrm{n} / \%)$ & $28 / 21.37 \%$ & $19 / 8.19 \%$ & $35 / 12.92 \%$ & $82 / 12.93 \%$ & $<0.002$ \\
Infection (n/\%) & $18 / 13.74 \%$ & $12 / 5.17 \%$ & $51 / 18.82 \%$ & $81 / 12.78 \%$ & $<0.001$ \\
Recurrence (n/\%) & $5 / 3.82 \%$ & $5 / 2.16 \%$ & $31 / 11.44 \%$ & $41 / 6.47 \%$ & $<0.001$ \\
\hline
\end{tabular}

concurrent abscess formation $(n=13)$. There were 509 males and 125 females, and the average age was $26.39 \pm 7.46$. The demographic data of the patients are shown in Table 1. There was not any significant difference between groups regarding gender $(p=0.74)$, age $(p=0.98)$ and removed specimen volume $(p=0.67)$. Days of hospitalization $(p=0.001)$ and operative time $(p=0.001)$ was shorter in midline closure group. The numbers of early postoperative complications (seroma, hematoma, wound separation, infection) and recurrences are summarized in Table 2. Recurrence rate $(p<0.001)$, infection rate $(p<0.001)$ and seroma rate $(p<0.001)$ was higher in the midline group than flap groups. Wound dehiscence rate was higher in the LTF group $(p<0.002)$.

\section{Discussion}

Management of pilonidal disease usually depends on the clinical presentation. While successful treatment has been achieved by shaving the hair on a regular basis, complex cases may require wide excision with extensive reconstructive procedures (Bailey et al., 2013; Spychala and Murawa, 2014). Surgery is usually preferred in the definitive treatment of pilonidal sinus disease. The mainstay of operative management for the chronic or persistent disease is excision of all pilonidal sinus tracts (Oncel et al., 2002; Petersen et al., 2002; Humphries and Duncan, 2010; Kepenekci, 2010). The optimal closure of the wound following an excision is debated. A close relationship has been reported between wound closure and postoperative morbidity and recurrence. Although many surgical techniques have been described for wound closure, most of these procedures fail to achieve the goals altogether. Primary closure can be accomplished by either midline or offmidline techniques including Z-plasty, V-Y advancement flap, Karydakis flap and Limberg flap (Bascom, 1987; Berkem et al., 2005).

It has been clearly stated in meta-analyses that off-midline closure should become standard management for pilonidal sinus when the closure is the desired surgical option (Omer et al., 2015). A systematic review and meta-analyses of randomized controlled trials including 1,573 patients showed benefits clearly with off-midline closure compared with midline closure (McCallum et al., 2008). 
In our series, we compared midline closure with off-midline closure techniques including KF and LTF. The mean age was $26.4 \pm 7$, and $80.2 \%$ of the patients were male. Recurrence rate (11.4\%), infection rate (18.8\%) and seroma rate $(15.1 \%)$ were higher in the midline group than flap groups $(\mathrm{p}<0.001)$. Only, hospitalization days $(1.7 \pm 1.5)$ and operative time $(27 \pm 6)$ were shorter in midline closure group. With the off-midline closure, the wound dehiscence incidence may be less developing due to less tension on the incision line, which may result in less recurrence than with midline closure. Also, because of the relatively high volume of specimens extracted in surgical flap techniques, drainage is used more often in these patients and therefore seroma may be less developing in off-midline closure techniques. In our study, it was observed that the specimen volumes obtained from off-midline closure patients were higher than those with midline closure, but there was no statistical difference between the two groups.

Karydakis (1992) reported a recurrence rate of $1 \%$, a complication rate of $8 \%$, and a length of hospital stay of 3 days. In our study, the recurrence rate was $2.2 \%$, seroma rate $4.3 \%$, infection rate $5.2 \%$, wound dehiscence rate $8.2 \%$ and length of hospital stay of $2.1 \pm 0.7$ days in KF group. Our results are consistent with the literature in this study.

Successful results regarding LTF have been reported in the literature. In a study, low complication and recurrence rate and higher postoperative quality of life have been published with LTF (Omer et al., 2015). In another study, a lower recurrence and lower complication rate with LTF compared to KF has been reported (Arslan et al., 2014). On the other hand, some authors have stated a high complication rate in LTF. A study has reported $49 \%$ total complication rate with seroma $6 \%$, wound dehiscence $45 \%$, skin necrosis $10 \%$, hematoma $6 \%$, infection $4 \%$ and recurrent disease 13\% (Käser et al., 2015). In the present study, the recurrence rate was 3.8\%, seroma rate $9.9 \%$, infection rate $13.7 \%$ and wound dehiscence rate $21.4 \%$ in LTF group. Wound dehiscence rate was higher (28 patients) in the LTF group. That may be due to surgical technique. Because the majority of wound dehiscence was not complete, and no major surgical intervention was required (the wound was sewn after cleaning) for these patients, but healing time was slightly longer.

Following surgical treatment of pilonidal disease, early postoperative morbidity and comfort is an important concern as well as recurrence. The most notable early postoperative problems are wound infection and wound dehiscence (Altintoprak et al., 2013; Bessa, 2013). In the present study, Karydakis flap group had $5.2 \%$ wound infection and $8.2 \%$ wound dehiscence while the LTF group had $13.7 \%$ wound infection and $21.4 \%$ wound separation. A significant difference was found between the groups $(p<0.001)$.

There are also conservative or minimally invasive treatment options in PSD. Common conservative treatment is the administration of phenol to the sinuses. A study reported that the best treatment option for pilonidal sinus disease was crystallized phenol treatment with laser depilation (Girgin et al., 2012). In another 
study, an innovative technique consisting in the destruction of the pilonidal cyst with a radial laser probe (FILACTM, Biolitec, Germany) has been described and concluded that the method could be proposed as a first-line treatment to the majority of patients with a pilonidal sinus disease (Dessily et al., 2017). In our opinion, the anatomic problem (narrow and deep natal cleft) which is an etiological factor in the development of PSD remains untreated in these techniques, and also recurrences rate is still high.

\section{Conclusion}

In this retrospective study, where we compare three widely used surgical methods in recent times, a significant difference was found between the techniques regarding early postoperative complications and recurrence risk. We believe that both offmidline closure methods can be safely used in PSD treatment, while KF group provides better results than LTF group regarding early postoperative complications. Midline closure method was superior regarding duration of surgery and hospitalization but has no advantage regarding early complications and recurrence.

\section{References}

Allen-Mersh, T. G. (1990) Pilonidal sinus: Finding the right tract for treatment. Br. J. Surg. 77, 123-132.

Altintoprak, F., Dikicier, E., Arslan, Y., Ozkececi, T., Akbulut, G., Dilek, O. N. (2013) Comparision of the Limberg flap with the $\mathrm{V}$-Y flap technique in the treatment of pilonidal disease. J. Korean Surg. Soc. 85 , 63-67.

Arslan, K., Said Kokcam, S., Koksal, H., Turan, E., Atay, A., Dogru, O. (2014) Which flap method should be preferred for the treatment of pilonidal sinus? A prospective randomized study. Tech. Coloproctol. 18(1), $29-37$.

Bailey, H. R., Billingham, R. P., Stamos, M. J., Snyder, M. J. (2013) Colorectal Surgery. Elsevier Saunders, Philadelphia.

Bascom, J. U. (1983) Pilonidal disease: long-term results of follicle removal. Dis. Colon Rectum 23, 800-807.

Bascom, J. U. (1987) Repeat pilonidal operations. Am. J. Surg. 154(1), 118-122.

Berkem, H., Topaloglu, S., Ozel, H., Avsar, F. M., Yildiz, Y., Yuksel, B. C., Hengirmen, S., Akyurek, N. (2005) V-Y advancement flap closures for complicated pilonidal sinus disease. Int. J. Colorectal Dis. 20(4), 343-348.

Bessa, S. S. (2013) Comparison of short-term results between the modified Karydakis flap and the modified Limberg flap in the management of pilonidal sinus disease: a randomized controlled study. Dis. Colon Rectum 56(4), 491-498.

Dessily, M., Charara, F., Ralea, S., Allé, J. L. (2017) Pilonidal sinus destruction with a radial laser probe: technique and first Belgian experience. Acta Chir. Belg. 117(3), 164-168.

Girgin, M., Kanat, B. H., Ayten, R., Cetinkaya, Z., Kanat, Z., Bozdağ, A., Turkoglu, A., Ilhan, Y. S. (2012) Minimally invasive treatment of pilonidal disease: crystallized phenol and laser depilation. Int. Surg. 97(4), 288-292.

Gurer, A., Gomceli, I., Ozdogan, M., Ozlem, N., Sozen, S., Aydin, R. (2005) Is routine cavity drainage necessary in Karydakis flap operation? A prospective, randomized trial. Dis. Colon Rectum 48(9), 1797-1799.

Hull, T. L., Wu, J. (2002) Pilonidal disease. Surg. Clin. North Am. 82(6), 1169-1185. 
Humphries, A. E., Duncan, J. E. (2010) Evaluation and management of pilonidal disease. Surg. Clin. North Am. 90, 113-124.

Karydakis, G. E. (1992) Easy and successful treatment of pilonidal sinus after explanation of its causative process. Aust. N. Z. J. Surg. 62(5), 385-389.

Käser, S. A., Zengaffinen, R., Uhlmann, M., Glaser, C., Maurer, C. A. (2015) Primary wound closure with a Limberg flap vs. secondary wound healing after excision of a pilonidal sinus: a multicentre randomised controlled study. Int. J. Colorectal Dis. 30(1), 97-103.

Kepenekci, I., Demirkan, A., Celasin, H., Gecim, I. E. (2010) Unroofing and curettage for the treatment of acute and chronic pilonidal disease. World J. Surg. 34(1), 153-157.

McCallum, I. J., King, P. M., Bruce, J. (2008) Healing by primary closure versus open healing after surgery for pilonidal sinus: systematic review and meta-analysis. BMJ 19, 868-871.

Omer, Y., Hayrettin, D., Murat, C., Mustafa, Y., Evren, D. (2015) Comparison of modified Limberg flap and modified elliptical rotation flap for pilonidal sinus surgery: a retrospective cohort study. Int. J. Surg. 16, 74-77.

Oncel, M., Kurt, N., Kement, M., Çolak, M., Eser, M., Uzun, H. (2002) Excision and marsupialization versus sinus excision for the treatment of limited chronic pilonidal disease: a prospective, randomized trial. Tech. Coloproctol. 6, 165-169.

Petersen, S., Koch, R., Stelzner, S., Wendlandt, T. P., Ludwig, K. (2002) Primary closure techniques in chronic pilonidal sinus: a survey of the results of different surgical approaches. Dis. Colon Rectum 45(11), 1458-1467.

Shabbir, F., Ayyaz, M., Farooka, M. W., Toor, A. A., Sarwar, H., Malik, A. A. (2014) Modified Limberg's flap versus primary closure for treatment of pilonidal sinus disease: a comparative study. J. Pak. Med. Assoc. 64(11), 1270-1273.

Shafik, A. (1996) Electrocauterization in the treatment of pilonidal sinus. Int. Surg. 81, 83-84.

Spychala, A., Murawa, D. (2014) The Limberg flap procedure in the treatment of pilonidal cyst disease of the sacrum - initial report. Pol. Przegl. Chir. 86(6), 257-262.

Varnalidis, I., loannidis, O., Paraskevas, G., Papapostolou, D., Malakozis, S. G., Gatzos, S., Tsigkriki, L., Ntoumpara, M., Papadopoulou, A., Makrantonakis, A., Makrantonakis, N. (2014) Pilonidal sinus: a comparative study of treatment methods. J. Med. Life 15, 27-30. 